\title{
The impact of HIV on presentation and outcome of bacterial sepsis and other causes of acute febrile illness in Gabon
}

\author{
Michaëla A. M. Huson · Rachel Kalkman · Sebastiaan M. Stolp • \\ Saskia Janssen · Abraham S. Alabi · Justin O. Beyeme • \\ Tom van der Poll $\cdot$ Martin P. Grobusch
}

Received: 17 September 2014 / Accepted: 16 February 2015 / Published online: 11 March 2015

(C) The Author(s) 2015. This article is published with open access at Springerlink.com

\begin{abstract}
Purpose HIV, bacterial sepsis, malaria, and tuberculosis are important causes of disease in Africa. We aimed to determine the impact of HIV on the presentation, causes and outcome of bacterial sepsis and other acute febrile illnesses in Gabon, Central Africa.

Methods We performed a prospective observational study in new adult admissions with fever or hypothermia ( $\geq 38$ or $<36^{\circ} \mathrm{C}$ ). Blood cultures, as well as HIV and malaria testing were performed in all patients.

Results We enrolled 382 patients, including 77 (20.2\%) with HIV infection. Malaria was the most frequent diagnosis $(n=130,34 \%)$, and was associated with a more severe presentation in HIV patients. Sepsis was also common $(n=107$, $28 \%$ ), including $29(7.6 \%)$ patients with culture confirmed bacterial bloodstream infection. Bacterial bloodstream
\end{abstract}

Electronic supplementary material The online version of this article (doi:10.1007/s15010-015-0753-2) contains supplementary material, which is available to authorized users.

M. A. M. Huson $(\square) \cdot$ T. van der Poll

Division of Infectious Diseases, Center of Experimental and Molecular Medicine, Academic Medical Center, University of Amsterdam, Meibergdreef 9, Room G2-105,

1105 AZ Amsterdam, The Netherlands

e-mail: m.a.huson@amc.uva.nl

M. A. M. Huson · R. Kalkman · S. M. Stolp · S. Janssen · M. P. Grobusch

Centre de Recherches Médicales de Lambaréné, Albert

Schweitzer Hospital, Lambaréné, Gabon

R. Kalkman · S. M. Stolp · S. Janssen · A. S. Alabi ·

J. O. Beyeme · M. P. Grobusch

Division of Infectious Diseases, Center of Tropical Medicine and Travel Medicine, Academic Medical Center, University of Amsterdam, Amsterdam, The Netherlands infections were more frequent in HIV patients, in particular with S. pneumoniae. Tuberculosis was observed in 29 (7.6\%) patients, and was also more common in HIV patients. The majority of HIV patients was newly diagnosed, and only 15 $(19.5 \%)$ were using combination antiretroviral therapy.

Conclusions Our findings illustrate the impact of HIV coinfection on the burden of sepsis, malaria and tuberculosis in Gabon, as well as the need to scale up HIV counseling, testing and treatment.

Keywords HIV $\cdot$ Sepsis $\cdot$ Bacteremia $\cdot$ Malaria $\cdot$ Fever . Tuberculosis

\section{Introduction}

The HIV epidemic is a major health problem in Africa that has changed the spectrum of acute illnesses, such as sepsis and malaria, in patients presenting to the hospital $[1,2]$. Sepsis is a heterogeneous condition that can be defined by the presence of clinical evidence for infection and at least two of four Systemic Inflammatory Response Syndrome (SIRS) criteria [3]. Sepsis is an important cause of morbidity and mortality worldwide, but detailed information on sepsis epidemiology in low- and middle income regions is largely unavailable. The immense burden of the HIV epidemic in Africa is likely to contribute to the number of sepsis cases in this region, as HIV patients are more likely to present with bacterial bloodstream infections (BSIs) [2]. Blood culture is an important diagnostic tool in the assessment of a septic patient, but microbiology laboratory facilities are often limited in developing regions. Therefore, gaining more insight into causative pathogens is vital to guide empirical antibiotic treatment. In Central Africa, studies on bacterial sepsis have been very limited, 
and no previous studies were performed in Gabon [4]. The HIV prevalence in adults in Gabon was estimated to be around $4 \%$ in 2012 [5], and likely influences the spectrum of disease in acutely ill patients, including bacterial BSIs and sepsis. Other major causes of febrile illness in Gabon include malaria which is endemic throughout the country and is predominantly caused by Plasmodium falciparum [6]. The prevalence of malaria might also be influenced by the HIV epidemic. In a systematic review, malaria was identified as the third cause of morbidity in HIV patients in Africa [7], and HIV infection is associated with an increased prevalence of symptomatic malaria, particularly in patients with severe immunosuppression [1]. In addition, tuberculosis is highly prevalent in central Africa [8], and is an important cause of morbidity and mortality in Gabon, particularly in HIV-positive patients [9].

With this study, we aimed to determine the influence of HIV infection on (1) patient characteristics, including factors related to health care-seeking behavior, and main diagnoses in newly admitted patients who present with signs of SIRS, (2) the prevalence of bacterial BSI, and causative pathogens in this patient population, and (3) patient outcome.

\section{Materials and methods}

\section{Setting}

Patients were recruited at the Albert Schweitzer Hospital (ASH) in Lambaréné, Gabon, from March 2012 until July 2013. The ASH is a 150-bed referral hospital in the Moyen Ogooué Province and its patients originate from throughout central Gabon.

\section{Study design and inclusion criteria}

We performed a prospective observational study. All new hospital admissions in adults (age $\geq 18$ years) who presented with fever or hypothermia (tympanic temperature $\geq 38$ or $<36{ }^{\circ} \mathrm{C}$ ), and at least one other SIRS criterion (tachycardia $>90 / \mathrm{min}$, respiratory rate $>20 / \mathrm{min}$ or a white blood cell count $<4 \mathrm{e} 9$ or $>12 \mathrm{e} 9 \mathrm{~g} / \mathrm{L}$ ) [10], were eligible for participation in the study. The study was conducted according to the principles of the Declaration of Helsinki in the current version of Seoul, 2008, and was approved by the scientific review committee of the "Centre des Recherches Medicales de Lambaréné". Written informed consent was obtained from all patients or their guardians prior to enrollment. Aerobic and anaerobic blood cultures, as well as HIV and malaria testing, were performed for all participating patients. For HIV-positive patients CD4 counts were also determined. Patients were included by the study physician during weekdays. In addition, blood culture bottles were made available to the hospital staff to perform blood cultures at night and during the weekend.

Clinical laboratory methods

Aerobic and anaerobic blood culture vials (Becton-Dickinson, Franklin Lakes, NJ, USA) were incubated in the automated BD Bactec 9050 system for a maximum of 5 days or until the culture became positive. Standard culture-based methods were used for species identification [API Test stripes (bioMérieux, Craponne, France) and BBL Enterotubes or BBL Oxi/Ferm Tube (Becton-Dickinson, Franklin Lakes, NJ, USA)]. Coagulase-negative staphylococci and Bacillus spp. were routinely considered contaminants. Streptococcus viridians were regarded contaminants as well, unless the patient had clinical signs of endocarditis or meningitis. As part of a clinical trial requirement, the microbiology laboratory at the ASH successfully participates in regular external quality assurance programs addressing species identification.

For HIV testing, a rapid test was used [Vikia HIV 1/2 (bioMérieux, Craponne, France), or Determine ${ }^{\mathrm{TM}}$ HIV 1/2, (Alere, Yavne, Israel), depending on local availability]. In case of a positive reading, the result was confirmed by VIDAS HIV DUO Ultra (bioMérieux, Craponne, France) and Immunocomb HIV1\&2 Bispot (Alere, Yavne, Israel). CD4 counts were done using BD FACS count (BectonDickinson, Franklin Lakes, NJ, USA). The Lambaréné method to analyze thick smears was applied to diagnose malaria [11].

\section{Case definitions}

Sepsis cases were defined by clinical evidence of infection combined with fever or hypothermia (tympanic temperature $\geq 38$ or $<36{ }^{\circ} \mathrm{C}$ ) and at least one other SIRS criterion. Infection diagnoses were classified on the basis of Centers for Disease Control and Prevention and International Sepsis Forum Consensus Conference definitions [12-14], adapted to the Gabonese situation where limited diagnostic tests were available (Online Resource 1: Case definitions for sites of infection in sepsis patients). Malaria cases were defined as Plasmodium spp. parasitemia (any parasite density) plus fever $\left(\geq 38.0^{\circ} \mathrm{C}\right)$ without another established cause of fever (blood culture negative). All patients who were treated with antituberculous drugs were classified as tuberculosis patients. In addition, for patients not (yet) on treatment, and for classification of the site of infection, we used the following definitions: Pulmonary tuberculosis was defined by the presence of at least two symptoms (chronic cough $>2$ weeks, night sweats, weight loss or fever) combined with a chest X-ray typical for pulmonary tuberculosis, a sputum positive smear by Ziehl-Neelsen or auramine staining. 
Extrapulmonary tuberculosis was identified by at least two symptoms (night sweats, weight loss or fever) combined with typical abnormalities on echography (including periportal or para-aortic lymphadenopathy, splenic microabscesses, ascites, pericardial or pleural effusion), on sight during surgical procedures or in cell compositions of specimens such as pleural or pericardial fluid, or by a positive Ziehl-Neelsen or auramine staining of an extrapulmonary site.

Statistical methods

Categorical variables are presented as percentages and continuous variables are presented as medians with their inter quartile range (IQR). A Kolmogorov-Smirnov test was used to determine the distribution of continuous variables. We used $\chi^{2}$ tests for comparisons of categorical variables, Mann-Whitney $U$ tests to assess differences in non-normally distributed continuous variables, and unpaired $t$ tests for normally distributed variables. For multivariate analyses of continuous variables, the Kruskal Wallis test was used for non-normally distributed variables, and one-way
ANOVA for normally distributed variables. A $p$ value of $<0.05$ was applied as level of significance in all analyses. Data analyses and creation of figures were done with SPSS statistics version 19 (IBM, Armonk, NY, USA) and GraphPad Prism (GraphPad Software, La Jolla, CA, USA).

\section{Results}

Patient characteristics and main diagnoses

We invited 405 eligible patients to participate in the study. Informed consent was obtained from 384 patients. Twentyone $(5.2 \%)$ patients refused participation to the study, the main reason for refusal being unwillingness to be tested for HIV. In two patients, blood draw was technically impossible, leaving 382 patients for inclusion in our analyses. Of 382 patients, 77 (20.2\%) were HIV positive (Online Resource 2: Study flowchart). Our patient population was relatively young (median age 34 years, IQR 25-46), with a female predominance $(n=240,63.8 \%)$ (Table 1$)$. HIV

Table 1 Characteristics of patients admitted to the ASH with acute febrile disease

\begin{tabular}{|c|c|c|c|c|c|}
\hline & Total $(n=382)$ & $\mathrm{HIV}+(n=77)$ & $\operatorname{HIV}-(n=305)$ & $p$ value & OR $(95 \% \mathrm{CI})$ \\
\hline \multicolumn{6}{|l|}{ Demographics } \\
\hline Age (years) & $34(25-46)$ & $37(31-45)$ & $33(23-47)$ & 0.04 & \\
\hline Male sex & $142(37.2)$ & $22(28.6)$ & $120(31.4)$ & 0.08 & $0.62(0.36-1.06)$ \\
\hline Duration of complaints (days) & $3(2-6)$ & $4(1-11)$ & $3(2-5)$ & $<0.0001$ & \\
\hline Travel time (h) & $0.5(0.3-1.0)$ & $0.5(0.5-1.6)$ & $0.5(0.3-1.0)$ & 0.005 & \\
\hline \multicolumn{6}{|l|}{ Diagnoses } \\
\hline Malaria & $130(34.1)$ & $14(18.2)$ & $116(38.0)$ & 0.001 & $0.36(0.19-0.68)$ \\
\hline Malaria parasites $/ \mu \mathrm{L}^{\mathrm{a}}$ & $7200(1560-24000)$ & $54000(21990-127200)$ & $5040(1425-18000)$ & 0.0003 & \\
\hline Sepsis & $107(28.1)^{\mathrm{b}}$ & $26(33.8)$ & $81(26.6)$ & 0.21 & $1.41(0.82-2.41)$ \\
\hline Blood culture positive sepsis & $30(7.9)^{\mathrm{b}}$ & $12(15.6)$ & $18(5.9)$ & 0.005 & $2.95(1.36-6.44)$ \\
\hline Abdominal infection ${ }^{\mathrm{b}}$ & $41(38.3)$ & $5(19.2)$ & $36(44.4)$ & 0.02 & $0.30(0.10-0.87)$ \\
\hline Pneumonia & $22(20.6)$ & $9(34.6)$ & $13(16.0)$ & 0.02 & 3.45 (1.23-9.66) \\
\hline Skin or soft tissue infection & $26(24.3)$ & $8(30.8)$ & $18(22.2)$ & 0.38 & $1.56(0.58-4.16)$ \\
\hline Urinary tract infection & $11(10.3)$ & $4(15.4)$ & $7(8.6)$ & 0.32 & $1.92(0.51-7.18)$ \\
\hline Primary bacteremia & $7(6.5)$ & $0(0.0)$ & $7(8.6)$ & 0.19 & $0.17(0.01-2.97)^{\mathrm{c}}$ \\
\hline Tuberculosis & $29(7.6)^{\mathrm{d}}$ & $17(22.1)$ & $12(3.9)$ & $<0.0001$ & $6.92(3.14-15.24)$ \\
\hline Pulmonary tuberculosis & $22(75.9)^{\mathrm{d}}$ & $13(76.5)$ & $9(75.0)$ & 0.92 & $1.08(0.19-6.06)$ \\
\hline Ziehl-Neelsen or auramine confirmed & $13(44.8)$ & $6(35.3)$ & $7(58.3)$ & 0.22 & $0.39(0.09-1.78)$ \\
\hline
\end{tabular}

For categorical variables, the absolute number is given with the percentage, and for continuous variables medians are given with their interquartile range. We used $\chi^{2}$ tests for comparisons of categorical variables, Mann-Whitney $U$ tests to assess differences for non-normally distributed continuous variables, and unpaired $t$ tests for normally distributed variables

All $p$ values and odds ratios that breached statistical significance $(p<0.05)$ are depicted in bold

$O R$ odds ratio, $C I$ confidence interval

a All malaria cases were caused by Plasmodium falciparum

b Abdominal infections include gastroenteritis, biliary tract infections, peritonitis, appendicitis, endometritis, and intra-abdominal abscesses

c 0.5 was added to each value to allow for calculation of an odds ratio

d Including two cases with both blood culture positive sepsis and pulmonary tuberculosis in one HIV-positive and one HIV-negative patient 
Table 2 Characteristics of HIVpositive patients admitted with acute febrile disease

For categorical variables, the absolute number is given with the percentage, and for continuous variables medians are given with their interquartile range. Multivariate analysis was done using the Kruskal-Wallis test

$c A R T$ combination antiretroviral therapy, $B S I$ bloodstream infection

\begin{tabular}{lcl}
\hline & HIV-positive patients $(n=77)$ & $p$ value \\
\hline HIV status known prior to admission & $35(45.5)$ & - \\
Patients with a history of cART & $21(27.3)$ & - \\
Patients on cART & $15(19.5)$ & - \\
CD4 count $(n=68)$ & $168(61-438)$ & 0.33 \\
CD4 count in patients on cART $(n=14)$ & $273(41-578)$ & \\
CD4 count in patients with no previous cART $(n=50)$ & $168(73-458)$ & 0.26 \\
CD4 count in patients who abandoned cART $(n=5)$ & $48(39-231)$ & \\
CD4 count in malaria cases $(n=13)$ & $377(121-730)$ & \\
CD4 count in sepsis cases $(n=22)$ & $168(95-398)$ & \\
CD4 count in culture confirmed BSI cases $(n=12)$ & $150(67-198)$ & \\
CD4 count in tuberculosis cases $(n=16)$ & $97(46-158)$ & \\
\hline
\end{tabular}

patients were slightly older than HIV-negative patients (37 years compared to 33 years, $p=0.04$ ), and had a longer duration of complaints prior to presentation [4 days (IQR $1-11)$ compared to 3 days (IQR $2-5$ ), $p \leq 0.0001]$. The majority of patients came from the vicinity of the hospital (median travel time $0.5 \mathrm{~h}$, IQR $0.3-1 \mathrm{~h}$ ), although patients with HIV tended to travel farther (median travel time $0.5 \mathrm{~h}$ (IQR 0.5-1.6 h). HIV patients presented more frequently with respiratory symptoms, like cough (39 (50.6\%) versus $79(25.9 \%)$ in HIV-negative patients $(p<0.0001))$ or shortness of breath $(29(37.7 \%)$ and $70(23.0 \%)$, respectively, $p<0.009)$ ), and weight loss (45 (58.4\%) versus $47(15.4 \%)$, respectively, $p<0.0001)$ ) (Online Resource 3: Clinical symptoms and signs of patients admitted to the Albert Schweitzer hospital with acute febrile illness). The vast majority of patients were included with fever (median temperature $38.9{ }^{\circ} \mathrm{C}\left(\mathrm{IQR} 38.6-39.0{ }^{\circ} \mathrm{C}\right)$; only $4(1.0 \%)$ patients had hypothermia on admission, and there were no differences in temperature according to HIV status. Chronic co-morbidities other than HIV infection at the time of admission were seen in 49 patients $(12.8 \%)$, the most important being hypertension $(n=26,6.8 \%)$, which was less common in HIV patients (1.3\% compared to $9.5 \%$, $p=0.02)$. Other co-morbidities included diabetes mellitus $(n=6,1.6 \%)$, and sickle cell disease $(n=4,1.0 \%)$.

Malaria was the most frequent diagnosis with 130 (34.1\%) cases (Table 1). HIV patients were less likely to present with malaria (18.2\% compared to $38.0 \%$, $p<0.0001)$, but when they had malaria, their parasitemia was higher $(54000 / \mu \mathrm{L}$ compared to $5040 / \mu \mathrm{L}, p=0.001)$. Sepsis was diagnosed in 107 (28.1\%) patients. Pneumonia was more frequent in HIV-positive patients (9 (34.4\%) compared to $13(16.0 \%)$ in HIV-negative patients, $p=0.02)$, while the reverse was true for abdominal infections (5 (19.2\%) versus 36 (44.4\%), respectively, $p=0.02)$. Other sites of infection were less frequent and were not differentially distributed according to HIV status.
Positive blood cultures were present in 29 (7.6\%) patients and were more often found in HIV-positive patients (15.6\% compared to $5.9 \%$, OR 2.95; $95 \%$ CI 1.36-6.44). We found no cases of bacterial BSI combined with Plasmodium parasitemia, but in two cases, one HIV-positive and one HIV-negative patient, a bacterial BSI was combined with pulmonary tuberculosis. As expected, tuberculosis was more frequently diagnosed in HIV-positive patients [22.1 versus $3.9 \%$ in HIV-negative patients (OR 6.92; $95 \%$ CI 3.14-15.24)].

The majority of HIV patients in our cohort were newly diagnosed with HIV during participation in the study ( $n=42,54.5 \%$ ) (Table 2). Among patients whose HIV status was already known, $21(27.3 \%)$ had a history of treatment with combination antiretroviral treatment (cART). However, six patients abandoned treatment, so at the time of admission only 15 patients $(19.5 \%)$ were on cART. CD4 counts were available for $68(88.3 \%)$ patients, and 47 (69.1\%) of these had advanced disease with CD4 counts below 350 cells $/ \mathrm{mm}^{3}$. Median CD4 count was 168 cells/ $\mathrm{mm}^{3}$ (IQR $61-738$ cells $/ \mathrm{mm}^{3}$ ) and did not differ between patients with or without cART, nor between various diagnostic categories (Table 2). The patients who abandoned treatment suffered from progressive disease with a median CD 4 count of 48 cells $/ \mathrm{mm}^{3}$ (IQR 39-231 cells $/ \mathrm{mm}^{3}$ ).

Data on health care-seeking behavior were available for $379(99.2 \%)$ patients (Table 3). The majority ( $n=295,77.8 \%$ ) had taken some form of medication for their current illness prior to presenting at the hospital, the most common being analgesics $(n=234$, $79.4 \%$ ), antimalarials ( $n=79,26.7 \%$ ), and antibiotics $(57,19.3 \%)$ (Table 3). Analgesics were more frequently used by patients without HIV infection, while antibiotics were taken more often by HIV-infected patients. The main sources of medication prior to hospitalization were pharmacies ( $n=93,32.7 \%$ ), hospitals ( $n=84,29.6 \%)$, and dispensaries $(n=36,12.7 \%)$. Patients with HIV 
Table 3 Health care-seeking behavior prior to hospitalization

\begin{tabular}{|c|c|c|c|c|c|}
\hline & Total $(n=379)$ & $\mathrm{HIV}+(n=77)$ & $\mathrm{HIV}-(n=302)^{\mathrm{a}}$ & $p$ value & OR $(95 \% \mathrm{CI})$ \\
\hline \multicolumn{6}{|c|}{ Medication for current illness ${ }^{b}$} \\
\hline Total & $295(77.8)$ & $56(72.7)$ & $242(80.1)$ & 0.1375 & $0.65(0.36-1.15)$ \\
\hline Analgesics & $234(79.4)$ & $35(62.5)$ & $200(82.3)$ & 0.0011 & $0.36(0.19-0.68)$ \\
\hline Antimalarials & $79(26.7)$ & $11(19.6)$ & $68(28.0)$ & 0.2408 & $0.63(0.31-1.29)$ \\
\hline Antibiotics & $57(19.3)$ & $20(35.7)$ & $37(15.2)$ & 0.0004 & $3.1(1.6-5.9)$ \\
\hline Other & $19(6.4)$ & $7(12.5)$ & $12(4.9)$ & 0.0365 & $2.75(1.03-7.34)$ \\
\hline \multicolumn{6}{|l|}{ Source of medication ${ }^{c}$} \\
\hline Pharmacy & $93(32.7)$ & $14(25.9)$ & $79(33.9)$ & 0.1927 & $0.63(0.32-1.25)$ \\
\hline Hospital & $84(29.6)$ & $26(46.4)$ & $58(24.9)$ & 0.0005 & $2.88(1.56-5.29)$ \\
\hline Other hospital & $55(19.4)$ & $18(32.1)$ & $37(15.9)$ & 0.0033 & $2.65(1.36-5.16)$ \\
\hline Study hospital & $29(10.2)$ & $8(14.3)$ & $21(9.0)$ & 0.1644 & $1.85(0.77-4.47)$ \\
\hline Dispensary & $36(12.7)$ & $7(12.5)$ & $28(12.4)$ & 0.8482 & $1.09(0.61-3.67)$ \\
\hline Traditional medicine & $5(1.8)$ & $1(1.8)$ & $3(1.3)$ & 0.7462 & $1.46(0.45-2.65)$ \\
\hline Various informal & $44(15.5)$ & $2(3.7)$ & $42(18.0)$ & 0.0085 & $0.17(0.04-0.75)$ \\
\hline
\end{tabular}

For categorical variables, the absolute number is given with the percentage, and for continuous variables medians are given with their interquartile range. We used $\chi^{2}$ tests for comparisons of categorical variables, Mann-Whitney $U$ tests to assess differences for non-normally distributed continuous variables, and unpaired $t$ tests for normally distributed variables

All $p$ values and odds ratios that breached statistical significance $(p<0.05)$ are depicted in bold

$O R$ odds ratio, $C I$ confidence interval

${ }^{a}$ Information on medication use prior to hospitalization was missing in 3 cases

b This includes only medication taken within 7 days prior to hospitalization

${ }^{c}$ Information on source of medication was available for 284 patients $(96.3 \%)$. Percentages in this part of the table are calculated using the number of cases for which data were available as denominator

infection had more often visited a hospital prior to presentation (OR 2.88; $95 \%$ CI 1.56-5.29), while HIV-negative patients made more use of informal sources of medication, including friends and local shops (OR 0.17; $95 \%$ CI 0.04-0.75). Notably, antimalarials and antibiotics were only obtained from formal health services like pharmacies, dispensaries and hospitals.

\section{Bacterial bloodstream infections}

In our prospective cohort of patients included by the study physician, culture confirmed true bacterial BSIs were present in 30 patients $(7.9 \%)$. An additional 84 blood cultures were taken by hospital staff in the absence of the study physician. Ten of these cultures yielded bacterial growth with a pathogenic organism (11.9\%), bringing the total number of bacterial isolates to 40 . The main isolates were Escherichia coli $(n=12,30.0 \%)$, Staphylococcus aureus ( $n=6,15.0 \%)$, and Streptococcus pneumoniae ( $n=5$, $12.5 \%$ ) (Table 4 ). When we stratified the causative pathogens according to HIV status, we found that $S$. pneumoniae was exclusively present in patients with HIV co-infection and this difference was statistically significant $(p=0.003)$. For other pathogens, no statistical differences were found.

\section{Patient outcome}

Median length of stay (LOS) in our cohort was 4 days (IQR 2-6) (Table 5). Malaria patients had the shortest duration of stay (3 days, IQR 3-4), sepsis patients stayed longer (5 days, IQR 3-8), and patients with tuberculosis had the longest LOS (13 days, IQR 9-14). The long duration of hospitalization in tuberculosis patients was due to the policy that patients should take the first ten days of antituberculous medication under supervision in the hospital. Overall mortality in our cohort was low ( $n=16,4.2 \%)$, as patients with malaria had only $0.8 \%$ mortality $(n=1)$. Patients with culture confirmed bacterial sepsis had the highest mortality of $17.2 \%(n=5)$. Patients with HIV co-infection had a longer LOS (median 5 days (IQR 3-9), compared to 4 days (IQR 3-5), $p=<0.0001$ ), and mortality was higher (13\% compared to $2 \%)$. When stratified according to the main diagnostic categories, LOS was significantly longer for HIV patients with malaria, but not for sepsis or tuberculosis. Mortality was higher in HIV patients in all diagnostic categories, but the difference did not reach statistical significance in the subgroup of culture proven sepsis patients and tuberculosis patients, most likely due to the small sample size of these groups. 
Table 4 Main causative pathogens of bacterial bloodstream infection and their distribution according to HIV status

\begin{tabular}{|c|c|c|c|c|c|}
\hline & Total $(n=40)$ & $\mathrm{HIV}+(n=15)$ & $\operatorname{HIV}-(n=23)$ & $p$ value & OR $(95 \% \mathrm{CI})$ \\
\hline Escherichia coli & $12(30.0)^{\mathrm{a}}$ & $3(20.0)$ & $8(34.8)$ & 0.33 & $0.47(0.10-2.16)$ \\
\hline Staphylococcus aureus & $6(15.0)^{\mathrm{a}}$ & $3(20.0)$ & $2(8.7)$ & 0.31 & $2.63(0.38-18.00)$ \\
\hline Streptococcus pneumoniae & $5(12.5)$ & $5(33.3)$ & $0(0.0)$ & 0.003 & $24.62(1.24-487.5)^{b}$ \\
\hline Salmonella typhi & $2(5.0)$ & $0(0.0)$ & $2(8.7)$ & 0.24 & $0.28(0.01-6.20)^{\mathrm{b}}$ \\
\hline Non-typhoidal salmonellae & $2(5.0)$ & $2(13.3)$ & $0(0.0)$ & 0.09 & $7.58(0.34-169.0)^{\mathrm{b}}$ \\
\hline Klebsiella pneumoniae & $1(2.5)$ & $0(0.0)$ & $1(4.3)$ & 0.41 & $0.48(0.02-12.68)^{\mathrm{b}}$ \\
\hline Serratia marcescens & $1(2.5)$ & $0(0.0)$ & $1(4.3)$ & 0.41 & $0.48(0.02-12.68)^{\mathrm{b}}$ \\
\hline Streptococcus viridans & $1(2.5)$ & $1(6.7)$ & $0(0.0)$ & 0.21 & $4.86(0.19-127.6)^{b}$ \\
\hline Neisseria meningitidis & $1(2.5)$ & $0(0.0)$ & $1(4.3)$ & 0.41 & $0.48(0.02-12.68)^{\mathrm{b}}$ \\
\hline Bacteroides spp. & $1(2.5)$ & $0(0.0)$ & $1(4.3)$ & 0.41 & $0.48(0.02-12.68)^{\mathrm{b}}$ \\
\hline B-Hemolytic streptococci ${ }^{\mathrm{c}}$ & $8(25.0)$ & $1(8.3)$ & $7(30.4)$ & 0.08 & $0.16(0.02-1.50)$ \\
\hline
\end{tabular}

For all variables, the absolute number is given with the percentage. $\chi^{2}$ tests were used for comparisons between HIV-positive and HIV-negative cases

All $p$ values and odds ratios that breached statistical significance $(p<0.05)$ are depicted in bold

$O R$ odds ratio, $C I$ confidence interval

a Including one case with an unknown HIV status

b 0.5 was added to each value to allow for calculation of an odds ratio

c Including group B (3), group C (3), and group D (2) streptococci. The single HIV-positive patient in this group was infected with a group C streptococci

Table 5 Patient outcome

\begin{tabular}{|c|c|c|c|c|c|}
\hline & Total $(n=382)$ & $\mathrm{HIV}+(n=77)$ & $\mathrm{HIV}-(n=305)$ & $p$ value & OR $(95 \% \mathrm{CI})$ \\
\hline \multicolumn{6}{|l|}{ Length of stay (days) } \\
\hline Total & $4(2-6)$ & $5(3-9)$ & $4(3-5)$ & $<0.0001$ & \\
\hline Malaria & $3(3-4)$ & $4(4-6)$ & $3(3-4)$ & 0.001 & \\
\hline Sepsis & $5(3-8)$ & $5(3-10)$ & $5(3-7)$ & 0.63 & \\
\hline Culture proven sepsis & $5(3-9)$ & $8(3-12)$ & $4(3-7)$ & 0.10 & \\
\hline Tuberculosis & $13(9-14)$ & $11(5-15)$ & $13(10-14)$ & 0.84 & \\
\hline \multicolumn{6}{|l|}{ In hospital mortality } \\
\hline Total & $16(4.2)$ & $10(13.0)$ & $6(2.0)$ & $<0.0001$ & $7.43(2.61-21.18)$ \\
\hline Malaria & $1(0.8)$ & $1(7.1)$ & $0(0.0)$ & 0.005 & $24.10(0.94-620.2)^{a}$ \\
\hline Sepsis & $7(6.5)$ & $4(15.4)$ & $3(3.7)$ & 0.04 & $4.73(0.98-22.73)$ \\
\hline Culture proven BSI & $5(17.2)$ & $3(25.0)$ & $2(11.8)$ & 0.35 & $2.50(0.35-17.95)$ \\
\hline Tuberculosis & $3(11.1)$ & $3(18.8)$ & $0(0.0)$ & 0.13 & $5.96(0.28-128.0)^{\mathrm{a}}$ \\
\hline
\end{tabular}

For categorical variables, the absolute number is given with the percentage, and for continuous variables medians are given with their interquartile range. We used $\chi^{2}$ tests for comparisons of categorical variables, Mann-Whitney $U$ tests to assess differences for non-normally distributed continuous variables, and unpaired $t$ tests for normally distributed variables

All $p$ values and odds ratios that breached statistical significance $(p<0.05)$ are depicted in bold

$O R$ odds ratio, $C I$ confidence interval, $B S I$ bloodstream infection

a 0.5 was added to each value to allow for calculation of an odds ratio

\section{Discussion}

We demonstrated that HIV co-infection has a major impact on the causes and outcome of acute febrile illness requiring hospitalization in Gabon. In malaria patients, HIV co-infection was associated with higher parasitemia, longer LOS in hospital, and higher mortality. In accordance, previous studies found increased severity of disease and impaired response to treatment in HIV-positive malaria patients [1]. This could be due to defects in the innate immune response to malaria, as natural killer, natural killer T, and $\gamma \delta \mathrm{T}$-cells, isolated from the peripheral blood of HIV patients, were recently shown to be less responsive to a challenge with $P$. falciparum parasites [15]. The prevalence of malaria 
in our cohort was high (34.1\%), and our figures are even likely to present an underestimation as use of antimalarial drugs prior to hospitalization was common. HIV infection was previously described as a risk factor for symptomatic malaria [1], so the HIV epidemic in Gabon may drive an increase in adult malaria cases. As malaria infection was even more frequent in HIV-negative patients (38\%), other factors are likely adding to the burden of malaria. Recent evidence indicates that malaria is an underrecognized driver of adult mortality in Africa [16], and one possibility is that the successful roll-out of malaria intervention strategies targeted at children and pregnant women has shifted the burden of disease to non-pregnant adults $[17,18]$.

Sepsis was an important reason for admission in HIVpositive patients $(33.8 \%$ ), and HIV-negative patients (26.6\%). In line with previous studies, HIV co-infection was associated with an increased prevalence of bacterial BSI in patients with sepsis, and enhanced sepsis-related mortality [2]. The relatively low overall prevalence of bacterial BSI in this cohort compared to that reported in a systematic review for African countries (7.6\% compared to $13.5 \%$ ) may be related to the fact that our population was relatively young and had few chronic co-morbidities. In accordance with previous studies [2, 4], we found $E$. coli, S. aureus and S. pneumoniae to be the main isolates in patients with BSI, and our study confirms an increased risk for S. pneumoniae BSI in HIV-positive patients, a pathogen that is known to frequently cause invasive disease in HIV patients $[4,19]$. Bloodstream infection with non-typhoidal Salmonella, a pathogen also known to be more common in patients with HIV co-infection [2, 20], was exclusively found in HIV-positive patients, but the number of cases was too small to demonstrate a significant difference with HIV-negative patients. Pathogenic mechanisms that may be related to increased prevalence and severity of bacterial sepsis were recently reviewed, and include defects in both innate and adaptive immunity [21].

Tuberculosis is an important cause of morbidity and mortality in HIV patients [22]. In accordance, we observed a higher prevalence of tuberculosis in HIV-positive patients ( $22.1 \%$ compared to $3.9 \%$ ). Key factors for the increased susceptibility of HIV patients to develop tuberculosis include general as well as Mycobacterium tuberculosis specific CD4 T cell depletion [22]. In comparison to a previous retrospective survey of tuberculosis in the same hospital, which registered an annual number of around 120 tuberculosis cases [9], the number of tuberculosis patients in this study is low $(n=29)$. A possible explanation for this difference is that the majority of hospitalized tuberculosis patients do not present with acute febrile illness, but rather are admitted with chronic complaints. The higher rate of HIV co-infection in tuberculosis patients in our cohort $(58.6 \%)$, compared to $28.5 \%$ in the previous study
[9], suggests that HIV patients with tuberculosis are more prone to present with an acute sepsis-like illness.

Non-prescription antibiotic use is common in Africa and is a driver of antimicrobial resistance [23]. In our cohort, $19.3 \%$ used antibiotics prior to hospitalization, but the majority of patients obtained their drugs from a previous visit to the hospital, indicating that the use of over-thecounter antibiotics is limited in Gabon. In HIV patients, the use of antibiotics for their current illness prior to hospitalization was higher, which was most likely related to increased severity and longer duration of illness. These patients were also more likely to have visited a hospital for their complaints prior to their hospitalization. Antimalarial drugs were more commonly used prior to hospitalization (26.7\% of cases with no difference according to HIV status) and were mostly bought in the pharmacy. These figures are low in comparison to other African countries, such as Nigeria, where a survey found $100 \%$ of antibiotics used were obtained without prescription [23]. The use of traditional medicine was previously reported to be very common in Gabonese patients with tuberculosis [24], and is also frequently observed in HIV patients in other African countries $[25,26]$. However, in our study the use of traditional medicine was limited to $1.8 \%$ of patients, with no differences according to HIV status. One explanation for the discrepancy is that traditional medicine is mostly used in the case of chronic illness, whereas our patients generally presented to the hospital with acute illness. In addition, patients may be reluctant to discuss the use of traditional medicine in a formal health care setting like the hospital.

Our data illustrate the burden of HIV infection in this region and gaps in HIV testing and treatment. The majority of HIV patients $(69.1 \%)$ presented with advanced disease with CD4 counts below 350 cells $/ \mathrm{mm}^{3}$, the current World Health Organization threshold for prioritizing the start of cART [27]. However, only $19.5 \%$ of HIV patients were using treatment at the time of admission. The need for increased availability of HIV counseling and testing is illustrated by the fact that more than half $(54.5 \%)$ of the HIV patients in our cohort were newly diagnosed. In addition, the finding that $20.2 \%$ of patients in our cohort were HIV positive illustrates that implementation of routine counseling and testing for hospitalized patients would be beneficial. In other African countries, provider-initiated testing and counseling proved to be very useful in increasing the number of patients tested and in reaching patients who perceived themselves to be at low risk [28, 29]. Although patient acceptance was variable in other settings [28], our study demonstrates a high level of acceptance for HIV testing among Gabonese patients. However, challenges exist in linking newly diagnosed patients to care and retaining them in care. Previous studies in Gabon and other settings demonstrated a high level of loss to follow-up in 
HIV patients and we also found that a significant proportion of patients who previously used treatment abandoned cART $(n=6,28.6 \%)$ [29].

There are some limitations of this study that need to be mentioned. First, due to limited availability of diagnostic tools, we were unable to provide a conclusive diagnosis for the entire cohort. Possible diagnoses in febrile patients who did not meet our criteria for malaria, sepsis or tuberculosis include viral illnesses, such as dengue or chikungunya, both of which have been previously reported in Gabon [30] as well as malaria episodes masked by previous treatment. In addition, as the study was not primarily designed to investigate tuberculosis, the indication for tuberculosis diagnostic tests was left to the treating physician. Tuberculosis was principally diagnosed by Ziehl Neelsen staining of sputum, or on clinical grounds, as alternative diagnostic means such as culture or PCR were not available. This may have resulted in an underestimation of the number of tuberculosis cases in this cohort. Third, we performed a hospital-based study, so our findings may not be representative for causes of morbidity and mortality in the community. Although the Albert Schweitzer Hospital is a regional referral hospital, the vast majority of patients came from the vicinity of the hospital, which suggests that patients in remote areas with limited infrastructure, particularly those with more severe disease, may not be able to reach the hospital.

\section{Conclusion}

In African countries like Gabon, the HIV epidemic has a major influence on the prevalence, severity and outcome of acute febrile illness due to sepsis, malaria or tuberculosis. This study is the first to systematically investigate bacterial BSIs and sepsis in Gabon, and illustrates the importance of microbiology laboratory facilities, in particular for HIV patients who present more frequently with bacterial BSI. In addition, we identified a large gap between the number of HIV patients in need of cART, and those who know their HIV status and receive proper treatment or care, representing an important challenge in the management of HIV in this region.

\begin{abstract}
Acknowledgments This work was supported by the foundation "De Drie Lichten" in The Netherlands. We acknowledge Becton-Dickinson (Becton-Dickinson, Franklin Lakes, NJ, USA) for providing blood culture bottles free of charge for this study.
\end{abstract}

Conflict of interest We declare that none of the authors have any conflicts of interest.

Open Access This article is distributed under the terms of the Creative Commons Attribution License which permits any use, distribution, and reproduction in any medium, provided the original author(s) and the source are credited.

\section{References}

1. Flateau C, Le Loup G, Pialoux G. Consequences of HIV infection on malaria and therapeutic implications: a systematic review. Lancet Infect Dis. 2011;11:541-56. doi:10.1016/ S1473-3099(11)70031-7.

2. Huson MA, Stolp SM, van der Poll T, Grobusch MP. Community-acquired bacterial bloodstream infections in HIV-infected patients: a systematic review. Clin Infect Dis. 2014;58:79-92. doi:10.1093/cid/cit596.

3. Wenzel RP. Treating sepsis. N Engl J Med. 2002;347:966-7. doi:10.1056/NEJMp020096.

4. Reddy EA, Shaw AV, Crump JA. Community-acquired bloodstream infections in Africa: a systematic review and metaanalysis. Lancet Infect Dis. 2010;10:417-32. doi:10.1016/ S1473-3099(10)70072-4.

5. HIV/AIDS JUNPo. Global report: UNAIDS report on the global AIDS epidemic. 2013.

6. Organization WH. World malaria report 2013.

7. Holmes CB, Losina E, Walensky RP, Yazdanpanah Y, Freedberg KA. Review of human immunodeficiency virus type 1-related opportunistic infections in sub-Saharan Africa. Clin Infect Dis. 2003;36:652-62. doi:10.1086/367655.

8. Janssen S, Huson MA, Belard S, Stolp S, Kapata N, Bates $\mathrm{M}$, et al. TB and HIV in the Central African region: current knowledge and knowledge gaps. Infection. 2014;42:281-94. doi:10.1007/s15010-013-0568-y.

9. Stolp SM, Huson MA, Janssen S, Beyeme JO, Grobusch MP. Tuberculosis patients hospitalized in the Albert Schweitzer Hospital, Lambarene, Gabon - a retrospective observational study. Clin Microbiol Infect. 2013;19:E499-501. doi:10.1111/1469-0691.12278.

10. Bone RC, Sibbald WJ, Sprung CL. The ACCP-SCCM consensus conference on sepsis and organ failure. Chest. 1992;101:1481-3.

11. Planche T, Krishna S, Kombila M, Engel K, Faucher JF, NgouMilama E, et al. Comparison of methods for the rapid laboratory assessment of children with malaria. Am J Trop Med Hyg. 2001;65:599-602.

12. Garner JS, Jarvis WR, Emori TG, Horan TC, Hughes JM. CDC definitions for nosocomial infections, 1988. Am J Infect Control. 1988;16:128-40.

13. Calandra T, Cohen J, International Sepsis Forum Definition of Infection in the ICUCC. The international sepsis forum consensus conference on definitions of infection in the intensive care unit. Crit Care Med. 2005;33:1538-48.

14. Horan TC, Andrus M, Dudeck MA. CDC/NHSN surveillance definition of health care-associated infection and criteria for specific types of infections in the acute care setting. Am J Infect Control. 2008;36:309-32. doi:10.1016/j.ajic.2008.03.002.

15. Finney CA, Ayi K, Wasmuth JD, Sheth PM, Kaul R, Loutfy $\mathrm{MR}$, et al. HIV infection deregulates innate immunity to malaria despite combination antiretroviral therapy. AIDS. 2013;27:32535. doi:10.1097/QAD.0b013e32835b3dfa.

16. Murray CJ, Rosenfeld LC, Lim SS, Andrews KG, Foreman KJ, Haring D, et al. Global malaria mortality between 1980 and 2010: a systematic analysis. Lancet. 2012;379:413-31. doi:10.1016/ S0140-6736(12)60034-8.

17. Bouyou-Akotet MK, Offouga CL, Mawili-Mboumba DP, Essola L, Madoungou B, Kombila M. Falciparum malaria as an emerging cause of fever in adults living in Gabon, Central Africa. BioMed Res Int. 2014;2014:351281. doi:10.1155/2014/351281.

18. Mharakurwa S, Mutambu SL, Mberikunashe J, Thuma PE, Moss WJ, Mason PR, et al. Changes in the burden of malaria following scale up of malaria control interventions in Mutasa District, Zimbabwe. Malar J. 2013;12:223. doi:10.1186/1475-2875-12-223. 
19. Gilks CF, Ojoo SA, Ojoo JC, Brindle RJ, Paul J, Batchelor BI, et al. Invasive pneumococcal disease in a cohort of predominantly HIV-1 infected female sex-workers in Nairobi, Kenya. Lancet. 1996;347:718-23.

20. Tabu C, Breiman RF, Ochieng B, Aura B, Cosmas L, Audi A, et al. Differing burden and epidemiology of non-Typhi Salmonella bacteremia in rural and urban Kenya, 2006-2009. PLoS One. 2012;7:e31237. doi:10.1371/journal.pone.0031237.

21. Huson MA, Grobusch MP, van der Poll T. The effect of HIV infection on the host response to bacterial sepsis. Lancet Infect Dis. 2015;15:95-108.

22. Zumla A, Raviglione M, Hafner R, von Reyn CF. Tuberculosis. N Engl J Med. 2013;368:745-55. doi:10.1056/NEJMra1200894.

23. Morgan DJ, Okeke IN, Laxminarayan R, Perencevich EN, Weisenberg S. Non-prescription antimicrobial use worldwide: a systematic review. Lancet Infect Dis. 2011;11:692-701. doi:10.1016/S1473-3099(11)70054-8.

24. Cremers AL, Janssen S, Huson MA, Bikene G, Bélard S, Gerrets RPM, et al. Perceptions, health care seeking behaviour and implementation of a tuberculosis control programme in Lambaréné, Gabon. Public Health Action. 2013;3:328-32.

25. Lubinga SJ, Kintu A, Atuhaire J, Asiimwe S. Concomitant herbal medicine and Antiretroviral Therapy (ART) use among HIV patients in Western Uganda: a cross-sectional analysis of magnitude and patterns of use, associated factors and impact on ART adherence. AIDS Care. 2012;24:1375-83. doi:10.1080/0954 0121.2011.648600.

26. Peltzer K, Preez NF, Ramlagan S, Fomundam H, Anderson J, Chanetsa L. Antiretrovirals and the use of traditional, complementary and alternative medicine by HIV patients in KwazuluNatal, South Africa: a longitudinal study. Afr J Tradit Complement Altern Med. 2011;8:337-45.

27. Organization WH. Consolidated guidelines on the use of antiretroviral drugs for treating and preventing HIV infection. 2013.

28. Roura M, Watson-Jones D, Kahawita TM, Ferguson L, Ross DA. Provider-initiated testing and counselling programmes in sub-Saharan Africa: a systematic review of their operational implementation. AIDS. 2013;27:617-26. doi:10.1097/ QAD.0b013e32835b7048.

29. Obermeyer CM, Bott S, Bayer R, Desclaux A, Baggaley R, Group MS. HIV testing and care in Burkina Faso, Kenya, Malawi and Uganda: ethics on the ground. BMC Int Health Hum Rights. 2013;13:6. doi:10.1186/1472-698X-13-6.

30. Leroy EM, Nkoghe D, Ollomo B, Nze-Nkogue C, Becquart P, Grard G, et al. Concurrent chikungunya and dengue virus infections during simultaneous outbreaks, Gabon, 2007. Emerg Infect Dis. 2009;15:591-3. doi:10.3201/eid1504.080664. 